\title{
The Mechanism of Bone Resorption Induced by Epiregulin, a Member of EGF Family
}

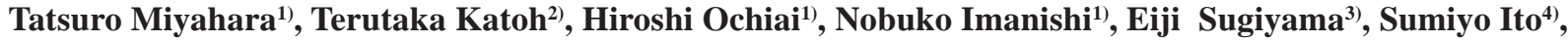 \\ Mitsunori Aono(), Yasutaro Mikami ${ }^{4)}$ Tsutomu Sakuma ${ }^{4)}$, Nobuo Nemoto ${ }^{4)}$ and Toshi Komurasaki ${ }^{5)}$
}

\begin{abstract}
1) Departments of Human Sience, ${ }^{2)}$ Public Health and ${ }^{3)}$ Internal Medicine, ${ }^{4)}$ Faculty of Medicine, and Department of Toxicology, Faculty of Pharmaceutical Sciences, Toyama Medical \& ${ }^{5)}$ Pharmaceutical University, and Molecular Biology Laboratory, Medicinal Research Laboratories, Taisho Pharmaceutical Co., Ltd.
\end{abstract}

\begin{abstract}
We investigated the effect of epiregulin (EPR) and EGF on the bone resorption using mice bones and cells. Both EPR and EGF enhanced bone resorption in ${ }^{45} \mathrm{Ca}$-prelabeled parietal bone without affecting osteoclast differentiation. These growth factors also enhanced the pit formation by femur-derived osteoclastrich fraction without increasing the number of the osteoclasts in a NS-398 (a COX-2 inhibitor)- sensitive manner. Furthermore, mature osteoclasts, differentiated from RANKL-stimulated RAW264.7 cells, induced pit formation with a longer time of EPR in a gefitinib (an EGFR tyrosinekinase inhibitor)-sensitive manner. These findings suggest that both EPR and EGF acted on osteoblasts to enhance bone resorption indirectly. In addition, EPR is suggested to have a direct stimulating pathway through the activation of EGFR on mature osteoclasts leading to bone resorption.
\end{abstract}

Key words : epiregulin, EGF, RANKL, osteoblast, osteoclast formation, osteoclast survival, pit formatioin, EGF receptor, RAW264.7 cell, Gefitinib (ZD1835)

\section{Introduction}

Epiregulin (EPR), a member of the epidermal growth factor (EGF) family, was purified from conditioned medium of mouse fibroblast-derived tumor cell line NIH-3T3/clone T7 cells. It is a 46-amino acid single chain peptide, and its amino acid sequence shows $24-50 \%$ amino acid sequence identity with sequence of other EGF-related growth factors. EPR exhibited bifunctional regulatory properties: it inhibited the growth of some types of cancer cells, but stimulated the growth of fibroblasts and various types of cells. EPR binds to EGFR and ErbB-4, but not to ErbB2 and ErbB-3, and induces tyrosine phosphorylation in four ErbB receptors by cross-talk. (T. Komurasaki et al. 1997). We have reported that a stimulative effect of EPR on the growth of primary mouse osteoblasts and an inhibitory effect of EPR on its differentiation are dependent on the activation of EGFR and p42/ 44 MAPK. EPR also stimulated bone resorption. In the present study, to clarify the mechanism of EPR-stimulated bone resorption, we investigated the effect of EPR on osteoclast formation, boneresorbing activity and survival of osteoclasts.

\section{Materials and Methods \\ Reagents \\ Monoclonal anti-phospho p42/44 MAPK antibody was purchased from Cell Signaling Technology. Polyclonal anti-EGFR antibody and anti-phosphotyrosine antibody (PY20) were obtained from Santa Cruz Biotechnologies. EGF, U0126, PD98059, NS98 and RANKL were obtained from Wako Pure Chemical Industries. Gefitinib (ZD1839) was a kind gift of Astra Zeneca (Macclesfield, UK).}

Cell Culture: A murine macrophage cell line RAW 264.7(RAW) (ATCC, Rockville, MD) was cultured on 9-cm diameter plastic dishes in DMEM containing 10\% FBS. For osteoclastogenesis assay, the cells were seeded on a 96-well plate in the presence or absence of RANKL and chemical reagents. After culture for the indicated time, the cells were fixed to stain the activity of tartrate- resistant acid phosphatase (TRAP). TRAP (+)-cells with three or more nuclei were counted as osteoclasts. To prepare RAW cellderived osteoclasts, RAW cells were cultured on the collagen gel with RANKL (50ng/ml) for 10 days and the osteoclasts were obtained by collagenase treatment. To examine EGFR expression in osteoclasts, RAW cells were cultured with 10?M U0160 and 10ng/ml RANKL for 3 days. After theformed osteoclasts were incubated with $100 \mathrm{ng} / \mathrm{ml}$ EPR or EGF for 10 min respectively, the cell lysates were prepared and used to detect phospho- EGFR by immnoblotting.

\section{Preparation of preexisting osteoclasts}

Suspension of preexisting osteoclasts were prepared by micing femora and tibiae of 13-day-old mice. To assay pit formation, the oteoclasts were added to each well of 96-well plate containing an ivory slice and cultured with the compounds for 4 days. After culture, the slice was stained with acid hematoxylin to detect resorbed pits.

Preparation of osteoclasts in co-culture of bone marrow cells and osteobalsts

Mouse bone marrow cells and osteoblasts were cultured on collagen gel with $10 \%$ FBS-MEM containing $10^{-8} \mathrm{M} 1,25(\mathrm{OH})_{2} \mathrm{D}_{3}$ and $10^{-6} \mathrm{M} \mathrm{PGE}_{2}$ for 7 days. To assay pit formation, crude osteoclasts obtained by collagenase treatment were cultured on ivoryslices for $24 \mathrm{hr}$. To assay osteoclast survival, crude osteoclasts were spotted on a 24-wellplate and treated with trypsin/EDTA to remove osteoblasts. Purified osteoclasts werecultured with EPR and/ or ZD1839 for 48 hr. and then stained with TRAP reagent.

\section{Results and Discussion}

EPR and EGF more than $1 \mathrm{ng} / \mathrm{ml}$ and above enhanced ${ }^{45} \mathrm{Ca}$ release from neonatal mouse parietal bones, in accordance with a previous study reporting that EGF enhanced bone resorption in a $\mathrm{PGE}_{2}$-dependent manner (A.H. Tashjian et al. 1978). Based on 
International symposium of Maxillofacial \& Oral Regenerative Biology in Okayama 2005

this finding, we investigated the possibility that this enhancement may occur due to a stimulatory osteoclast formation and/or a stimulatory bone-resorbing activity. As a result, after a disappearance of preexisting osteoclasts the long bones of 13day-old mice, treatment with $1,25(\mathrm{OH})_{2} \mathrm{D}_{3}$ induced reappearance of a newly formed osteoclasts. However EPR and EGF did not induce them. In RAW 264.7(RAW) cells, RANKL (osteoclast differentiation factor) enhanced osteoclast formation but EPR and EGF also did not enhance it. A level of p42/44 MAPK phosphorylation in EPR-treated RAW cells were much higher than that in the untreated cells. PD98059 and U0126, MEK inhibitors, enhanced the differentiation of RAW cells into osteoclasts in the presence of RANKL (10ng/ml), which alone did not induce osteoclast formation. Activation of p42/44 MAPK by RANKL was significantly inhibited by U0126 in these osteoclasts. Taken together, EPR-induced activation of p42/44 MAPK may cause a lack of response to osteoclast formation.

Next, a bone-resorbing activity of preexisting osteoclasts was evaluated by a pit formation assay, in which the osteoclasts were cultured on ivory slices. Both EPR and EGF as well as $1,25(\mathrm{OH})_{2} \mathrm{D}_{3}$ enhanced the pit formation and this pit-forming activity was inhibited by elcatonin. Both growth factors did not affect the number of osteoclasts on the slices, which was not decreased by elcatonin. These results suggested that EPR and EGF enhanced the pit formation through the activation of osteoclasts without increasing the number of osteoclasts.

Pit formation by crude osteoclasts prepared in the co-culture of mouse bone marrow cells and ostoblasts was enhanced by EPR and EGF. This enhancement was inhibited by NS398, a COX-2 inhibitor, and Gefitinib (ZD1839), an EGFR tyrosine kinase inhibitor.

Because the expression of COX-2 and EGFR is recognized in osteoblasts, EPR and EGF may stimulate osteoclastic bone resorption indirectly through osteoblast activation.

Because osteoclast function is activated by osteoblasts through a mechanism involving cell-to-cell and/or cell-to matrix contact (E. Jimi et al. 1996), we used RAW cell- derived osteoclasts containing no osteoblasts, which were prepared as described above. EPR as well as RANKL enhanced pit formation and its enhancement was inhibited by ZD1839. These findings suggest that EPR acts directly on osteoclasts to enhance bone resorption in a ZD1839-sensitive manner.

In addition, crude osteoclasts obtained from the co-culture were spotted on 24-well plate and treated with trypsin/EDTA to remove osteoblasts. When EPR and/or ZD1839 were cultured with purified osteoclasts for $48 \mathrm{hr}$, EPR enhanced osteoclast survival and its enhancement was depressed by ZD1839. This result indicates that EPR acts directly on osteoclasts alone and enhances osteoclast survival in a ZD1839-sensitive manner.

To examine the EGFR expression in osteoclasts, we prepared RAW cell-derived osteoclasts, which were treated with EPR or EGF $(100 \mathrm{ng} / \mathrm{ml})$ for $10 \mathrm{~min}$. and detected p-EGFR by western blot analysis. EPR and EGF were found to phosphorylate EGFR, which was recognized in A431 cells, a highly expressed cells of EGFR. It was shown thatEPR and EGF directly acted on mature osteoclasts through the activation of EGFR. .

In summary, EPR and EGF did not stimulate osteoclast differentiation. This finding leads to an assumption that osteoclast formation shows no contribution to EPR- and EGF-mediated bone resorption. EPR and EGF act on osteoblasts to stimulate osteoclastic bone reorption indirectly. In addition, we suggest that EPR and EGF have adirect stimulating pathway through the activation of EGFR on mature osteoclasts leading to bone resorption. 